\title{
Comparison of genomic and antimicrobial resistance features of latex agglutination test-positive and latex agglutination test-negative Staphylococcus aureus isolates causing bovine mastitis
}

\author{
A. Moser, ${ }^{*}$ R. Stephan, ${ }^{*}$ S. Corti, ${ }^{*}$ and S. Johler ${ }^{*} \dagger^{1}$ \\ *Institute for Food Safety and Hygiene, Vetsuisse Faculty, University of Zurich, Winterthurerstrasse 272, 8057 Zurich, Switzerland \\ †Skirball Institute of Biomolecular Medicine, New York University Medical Center, 540 First Avenue, New York 10016
}

\begin{abstract}
The dairy industry suffers massive economic losses due to staphylococcal mastitis in cattle. The Staphaureux latex agglutination test (Oxoid, Basel, Switzerland) was reported to lead to negative results in $54 \%$ of bovine Staphylococcus aureus strains, and latex-negative strains are thought to be less virulent than Staphaurex latex-positive strains. However, comparative information on virulence and resistance profiles of these 2 groups of Staph. aureus is scarce. Our objective was to associate the latex agglutination phenotype of Staph. aureus strains isolated from bovine mastitis milk with data on clonal complexes, virulence genes, and antibiotic resistance to (1) determine the virulence profiles of the Staphaureux test positive and Staphaurex test negative groups, and (2) provide data needed to improve treatment of bovine mastitis and to identify potential vaccine targets. Seventy-eight Staph. aureus strains isolated from 78 cows on 57 Swiss farms were characterized. Latex agglutination was tested by Staphaureux kit, and resistance profiles were generated by disk diffusion. A DNA microarray was used to assign clonal complexes $(\mathrm{CC})$ and to determine virulence and resistance gene profiles. By the Staphaureux test, $49 \%$ of the isolates were latex-positive and $51 \%$ were latex-negative. All latex-negative strains were assigned to CC151, whereas latex-positive strains were assigned to various clonal complexes, including CC97 $(\mathrm{n}=16)$, CC8 (n = 10), CC479 ( $\mathrm{n}=5), \operatorname{CC} 20(\mathrm{n}=4), \mathrm{CC} 7$ $(\mathrm{n}=1)$, CC9 $(\mathrm{n}=1)$, and CC45 $(\mathrm{n}=1)$. Although the latex-negative isolates were susceptible to all antimicrobial agents tested, $24 \%$ of latex-positive isolates were classified as intermediate with regard to cefalexinkanamycin and $13 \%$ were resistant to both ampicillin and penicillin. Microarray profiles of latex-negative isolates were highly similar, but differed largely from
\end{abstract}

Received July 15, 2012.

Accepted September 18, 2012.

${ }^{1}$ Corresponding author: sophia.johler@uzh.ch those of latex-positive isolates. Although the latex-negative group lacked several enterotoxin genes and $s a k$, it exhibited significantly higher prevalence rates of genes encoding enterotoxin $\mathrm{C}$, toxic shock syndrome toxin, and leukocidins (lukM/lukF-P83, lukD). Our findings suggest that latex-negative isolates represent a group of closely related strains with specific resistance and virulence gene patterns.

Key words: Staphylococcus aureus, bovine mastitis, Staphaurex latex agglutination test, virulence

\section{INTRODUCTION}

The dairy industry suffers from massive economic losses due to staphylococcal mastitis in cattle (Wells et al., 1998). The Staphaurex latex agglutination test (Oxoid, Basel, Switzerland) is a diagnostic instrument widely used to confirm putative Staphylococcus aureus isolates through detection of characteristic Staph. aureus surface proteins. Latex particles coated with human IgG and fibrinogen interact with the bacterial target proteins SpA (staphylococcal protein A), ClfA/B (clumping factor A/B), and FnbA/B (fibronectin-binding protein $\mathrm{A} / \mathrm{B}$ ), mediating a rapid agglutination reaction that is visible to the naked eye. Although the Staphaurex latex agglutination test exhibits high specificity (99.5\%) and sensitivity (99.8\%) when applied to Staph. aureus strains obtained from humans, Stutz et al. (2011) reported that $54 \%$ of Staph. aureus isolates obtained from cases of bovine mastitis yield negative test results. These false-negative results are due to sequence polymorphisms leading to impaired functionality of one or several of the targeted virulence factors (SpA, ClfA/B, or FnbA/B). Therefore, Staphaurex latex agglutination test (SLAT)-negative [SLAT $(-)]$ strains are thought to be less virulent than SLAT-positive [SLAT(+)] strains (Stutz et al., 2011). Although assessing the virulence potential of SLAT $(-)$ strains is of crucial importance to the dairy industry, data on the genomic background and antimicrobial resistance of bovine SLAT(-) isolates are scarce. 
Although antibiotic treatment is widely used to fight bovine mastitis, its merits are controversial. Use of antimicrobial agents is not only economically questionable and favors the development of antibiotic resistance, but it is also unsuitable in addressing intracellular persistence of the organism (Steeneveld et al., 2011; Fluit, 2012; Saini et al., 2012). Therefore, increased efforts are now focused on the development of vaccines. Recent studies postulate extended characterization of the genetic background of bovine mastitis isolates to enable identification of proteins crucial for colonization and infection that could serve as biomarkers in the identification of vaccine targets (Fluit, 2012; Klein et al., 2012).

The objective of this study was to link the latex agglutination phenotype of Staph. aureus strains isolated from bovine mastitis milk with data on clonal complexes, virulence genes, and antibiotic resistance to (1) determine the virulence profiles of the SLAT $(+)$ and SLAT(-) groups, and (2) provide data needed to improve treatment of bovine mastitis and to identify potential vaccine targets.

\section{MATERIALS AND METHODS}

\section{Bacterial Isolates, DNA Extraction, and Presumptive Species Identification}

Seventy-eight Staph. aureus strains were isolated from bovine mastitis milk samples collected from different cows on 57 Swiss farms between March 2011 and February 2012. Putative Staph. aureus isolates were identified by streaking samples onto rabbit plasma fibrinogen plates (Oxoid), which were subsequently incubated at $37^{\circ} \mathrm{C}$ and examined for coagulase activity after 48 h. A single typical Staph. aureus colony from each plate was transferred to blood agar and incubated overnight at $37^{\circ} \mathrm{C}$. Then, DNA was isolated using kits supplied by Qiagen (Hilden, Germany), according to the manufacturer's instructions. The concentration of nucleic acids was measured by using a Nanodrop ND1000 UV/Vis spectrophotometer (NanoDrop Technologies, Wilmington, DE).

\section{Staphaureux Latex Agglutination Test and Genotyping}

The Staphaurex test kit (Oxoid) was used according to the manufacturer's instructions to determine latex agglutination.

The presence of 284 genes and allelic variants was assessed using StaphyType ArrayStrips (Clondiag Chip Technologies, Jena, Germany) following the manufacturer's instructions. Multiplex linear DNA amplification and microarray hybridization allowed for identifi- cation of species markers, genes conferring resistance to antimicrobial agents, and virulence determinants such as genes encoding enterotoxins, toxic shock syndrome toxin, leukocidins, hemolysins, and adhesins. The microarray also enables assignment of strains to clonal complexes and agr types. The DNA microarray profiles were converted to sequence-like strings, as described elsewhere, to allow for visualization by SplitsTree4 (http://www.splitstree.org/), a software package designed to compute unrooted phylogenetic networks from molecular sequence data (Wattinger et al., 2012).

\section{Susceptibility Testing}

Disk diffusion was used to classify isolates as susceptible, intermediate, or resistant depending on respective zone diameters following Clinical and Laboratory Standards Institute (CLSI) standard protocols (CLSI, 2008). All antimicrobial agents were chosen with regard to their relevance in mastitis therapy. Antibiotic agents tested included ampicillin $(30 \mu \mathrm{g})$, amoxicillin $(20 \mu \mathrm{g})$ with clavulanic acid $(10 \mu \mathrm{g})$, cephalothin $(30 \mu \mathrm{g})$, ceftiofur $(30 \mu \mathrm{g})$, erythromycin $(15 \mu \mathrm{g})$, cefoxitin $(30 \mu \mathrm{g})$, gentamicin $10(\mu \mathrm{g})$, kanamycin $(30 \mu \mathrm{g})$, kanamycincefalexin $(30 \mu \mathrm{g}-15 \mu \mathrm{g})$, penicillin (10 IU), and penicillin-novobiocin (10 IU-30 $\mu \mathrm{g})$. Mueller-Hinton agar and disks containing ceftiofur and penicillin-novobiocin were provided by Oxoid, and disks containing cefalexinkanamycin (Ubrolexin) were provided by Boehringer Ingelheim (Basel, Switzerland). All other disks containing antibiotic agents were obtained from Becton Dickinson (Basel, Switzerland). Reference strain Staph. aureus ATCC 25923 was used as a quality control.

\section{Statistical Analysis}

The distribution of specific genes among latex-positive and latex-negative isolates was compared based on the hybridization results of the DNA microarray. SPSS Statistics 19 (SPSS Inc., Chicago, IL) was used to run the Pearson $\chi^{2}$ test, identifying significant associations between the latex phenotype and the presence of the examined genes. Results were considered to be statistically significant for $P$-values $<0.05$.

\section{RESULTS}

\section{Species Confirmation and Exclusion Criteria}

All isolates were confirmed to represent Staph. aureus using the species markers of the DNA microarray. To avoid bias, the sample collection was screened for identical isolates by comparison of all features tested, including microarray profiles and resistance patterns, and all isolates were found to be unique. 


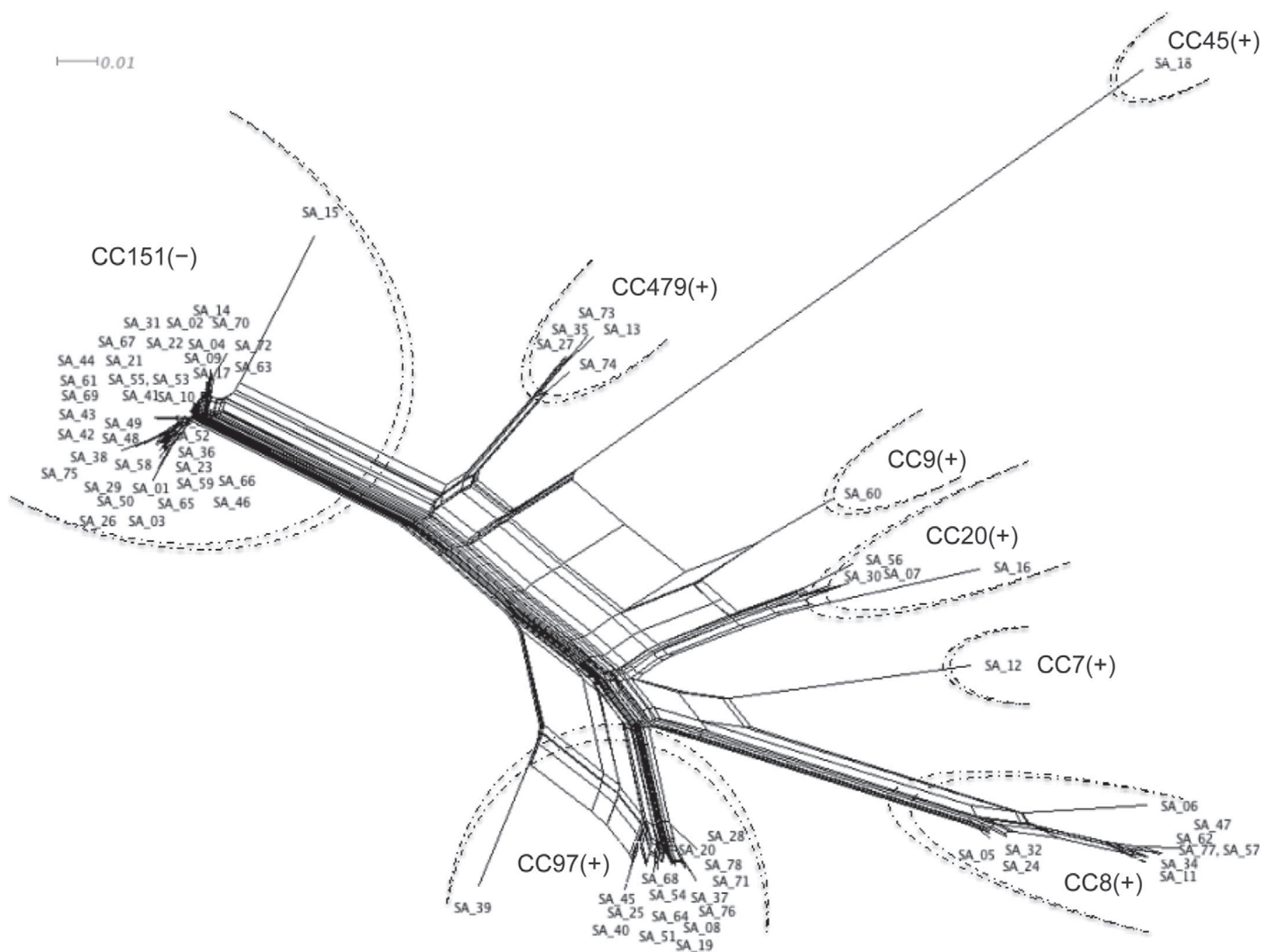

Figure 1. A SplitsTree (http://www.splitstree.org; Huson and Bryant, 2006) phylogenetic tree depicting similarity of resistance and virulence gene profiles among isolates testing positive [SLAT $(+)$ ] and negative [SLAT $(-)$ ] by the Staphaurex latex agglutination test (Oxoid, Basel, Switzerland). A unique identifier was assigned to each Staphylococcus aureus isolate (e.g., SA_01). The latex phenotype (+ or -) of all isolates clustered in one clonal complex is indicated in parentheses; clonal cluster (CC) 151 represents the only cluster comprising SLAT(-) isolates.

\section{Latex Agglutination and Clonal Complexes}

Latex agglutination was tested for all 78 confirmed bovine Staph. aureus strains. Although 38 isolates (49\%) were latex-positive and would have been correctly identified as Staph. aureus, 40 isolates (51\%) were latex-negative (i.e., false negatives). As depicted in the phylogenetic tree in Figure 1, all latex-negative strains exhibited very similar resistance and virulence gene profiles. They formed a single cluster of isolates assigned to clonal complex (CC) 151, whereas latexpositive strains were assigned to various clusters, including CC97 ( $\mathrm{n}=16), \mathrm{CC} 8(\mathrm{n}=10), \mathrm{CC} 479(\mathrm{n}=5)$, CC20 (n = 4), CC7 (n=1), CC9 $(\mathrm{n}=1)$, and CC45 $(\mathrm{n}=1)$.

\section{Resistance Phenotypes (Disk Diffusion)}

Resistance phenotypes determined by disk diffusion are listed in Table 1 . The SLAT $(-)$ isolates were susceptible to all antimicrobial agents tested. Among the SLAT (+) group, $24 \%$ of isolates were classified as intermediate with regard to cefalexin-kanamycin, and $13 \%$ of isolates were classified as resistant to both ampicillin and penicillin. No methicillin-resistant Staph. aureus (MRSA) isolates were detected among the bovine mastitis strains investigated in this study.

\section{Resistance and Virulence Gene Profiles}

Selected DNA microarray results on resistance and virulence genes are depicted in Table 2. Although no 
Table 1. Antimicrobial resistance phenotypes determined by disk diffusion among isolates testing positive [SLAT $(+)]$ or negative [SLAT $(-)]$ by the Staphaurex latex agglutination test (SLAT; Oxoid, Basel, Switzerland)

\begin{tabular}{|c|c|c|c|c|c|c|c|c|c|c|c|}
\hline $\begin{array}{l}\text { SLAT status/ } \\
\text { clonal complex }\end{array}$ & \multicolumn{11}{|c|}{ Antimicrobial agent ${ }^{1}$} \\
\hline \multicolumn{12}{|l|}{$\operatorname{SLAT}(+)$} \\
\hline $\mathrm{CC} 7(\mathrm{n}=1)$ & 1 & 0 & 0 & 0 & 0 & 0 & 0 & 0 & 0 & 1 & 0 \\
\hline CC8 $(\mathrm{n}=10)$ & 1 & 0 & 0 & 0 & 0 & 0 & 0 & 0 & $(1)^{2}$ & 1 & 0 \\
\hline $\mathrm{CC} 45(\mathrm{n}=1)$ & 1 & 0 & 0 & 0 & 0 & 0 & 0 & 0 & 0 & 1 & 0 \\
\hline $\operatorname{CC} 97(\mathrm{n}=16)$ & 1 & 0 & 0 & 0 & 0 & 0 & 0 & 0 & $(4)^{2}$ & 1 & 0 \\
\hline $\mathrm{CC} 479(\mathrm{n}=5)$ & 0 & 0 & 0 & 0 & 0 & 0 & 0 & 0 & 0 & 0 & 0 \\
\hline Total $(\mathrm{n}=78)$ & 5 & 0 & 0 & 0 & 0 & 0 & 0 & 0 & $(9)^{2}$ & 5 & 0 \\
\hline
\end{tabular}

${ }^{1} \mathrm{AM}=$ ampicillin, $\mathrm{AMC}=$ amoxicillin with clavulanic acid, $\mathrm{CF}=$ cephalothin, EFT $=$ ceftiofur, ERY = erythromycin, FOX $=$ cefoxitin, GM = gentamicin, $\mathrm{K}=$ kanamycin, $\mathrm{K} / \mathrm{CFX}=$ kanamycin-cefalexin, $\mathrm{P}=$ penicillin, $\mathrm{P} / \mathrm{NB}=$ penicillin-novobiocin.

${ }^{2}$ The number of isolates that exhibited intermediate sensitivity to the respective antimicrobial agent is presented in parentheses.

resistance genes were detected among $\operatorname{SLAT}(-)$ isolates, some $\operatorname{SLAT}(+)$ isolates exhibited genes involved in resistance to antibiotic agents, including $b l a I / R / Z$ (26\%), ermC (3\%), fosB (39\%), and vanB (3\%). Several SLAT $(+)$ strains also displayed the enterotoxin genes entA (21\%), entD (26\%), and entJ (16\%), as well as sak, encoding staphylokinase $(26 \%)$, virulence factors that were not found among $\operatorname{SLAT}(-)$ isolates. In contrast, the SLAT $(-)$ group exhibited significantly higher prevalence rates of both tested allelic variants of entC, as well as the egc enterotoxin gene cluster, genes encoding toxic shock syndrome toxin, and the leukocidins $l u k M / l u k F-P 83$ and $l u k D$. Neither $p v l$, encoding panton-valentine leukocidin, nor et $A / B / C$, encoding exfoliative toxins, was detected in this study. Microarray data on the presence of selected adhesin genes coding for target proteins of the Staphaureux test are presented in Table 2. Although almost all strains were positive for one or more allelic variants of the adhesin genes tested, latex-positive and latex-negative isolates differed largely in the allelic variants found. A comprehensive overview of the prevalence rates of all genes tested is provided as a supplemental file (available at http://www.journalofdairyscience.org/) that includes $P$-values used to determine significant differences in the prevalence of respective genes among SLAT $(+)$ and $\operatorname{SLAT}(-)$ isolates.

\section{DISCUSSION}

Although the Staphaureux test kit exhibits high specificity (99.5\%) and sensitivity (99.8\%) when applied to Staph. aureus strains obtained from humans, it frequently leads to false-negative results when applied to Staph. aureus isolates obtained from bovine masti- tis milk. In this study, $51 \%$ of isolates were SLAT(-), consistent with the rate of $54 \%$ reported by Stutz et al. (2011). In both Stutz et al. (2011) and the current study, SLAT $(+)$ isolates were associated with a wide range of clonal complexes, whereas the SLAT(-) isolates were assigned to CC151 only. Clonal complex 151 is reported to represent the most prevalent clonal complex among Staph. aureus isolated from bovine milk obtained from both seemingly healthy and clinically infected udders (Sakwinska et al., 2011; Stutz et al., 2011; Schlotter et al., 2012). The most frequent clonal complexes among SLAT $(+)$ strains in our study included CC97, CC8, CC497, and CC20, whereas CC7, $\mathrm{CC} 9$, and $\mathrm{CC} 45$ were assigned to only one isolate each. Interestingly, no isolates were assigned to CC133, a dominant clonal complex among Staph. aureus isolated from the milk of seemingly healthy cows in Germany (Schlotter et al., 2012).

When antimicrobial susceptibility was determined, the resistance proportions among clonal complexes differed, with CC151, CC479, and CC20 representing the only clonal complexes that included no resistant isolates. These findings are consistent with an extensive study on antimicrobial resistance among Swiss and French bovine Staph. aureus isolates, in which penicillin resistance rates among $\mathrm{CC} 151$ and $\mathrm{CC} 20$ were found to be far lower than those among CC97 (Sakwinska et al., 2011). Overall, 6\% of bovine Staph. aureus isolates tested in our study were resistant to both ampicillin and penicillin, and $12 \%$ exhibited intermediate susceptibility to kanamycin/cefalexin. Although the emergence of livestock-associated MRSA represents an increasing problem in Switzerland (Huber et al., 2010) and worldwide, no MRSA isolates were detected among the isolates investigated in our study. The single strain 
Table 2. Prevalence (\%) of selected virulence and resistance genes detected by DNA microarray among bovine mastitis isolates testing positive $[\operatorname{SLAT}(+)]$ and negative [SLAT(-)] by the Staphaurex latex agglutination test (SLAT; Oxoid, Basel, Switzerland)

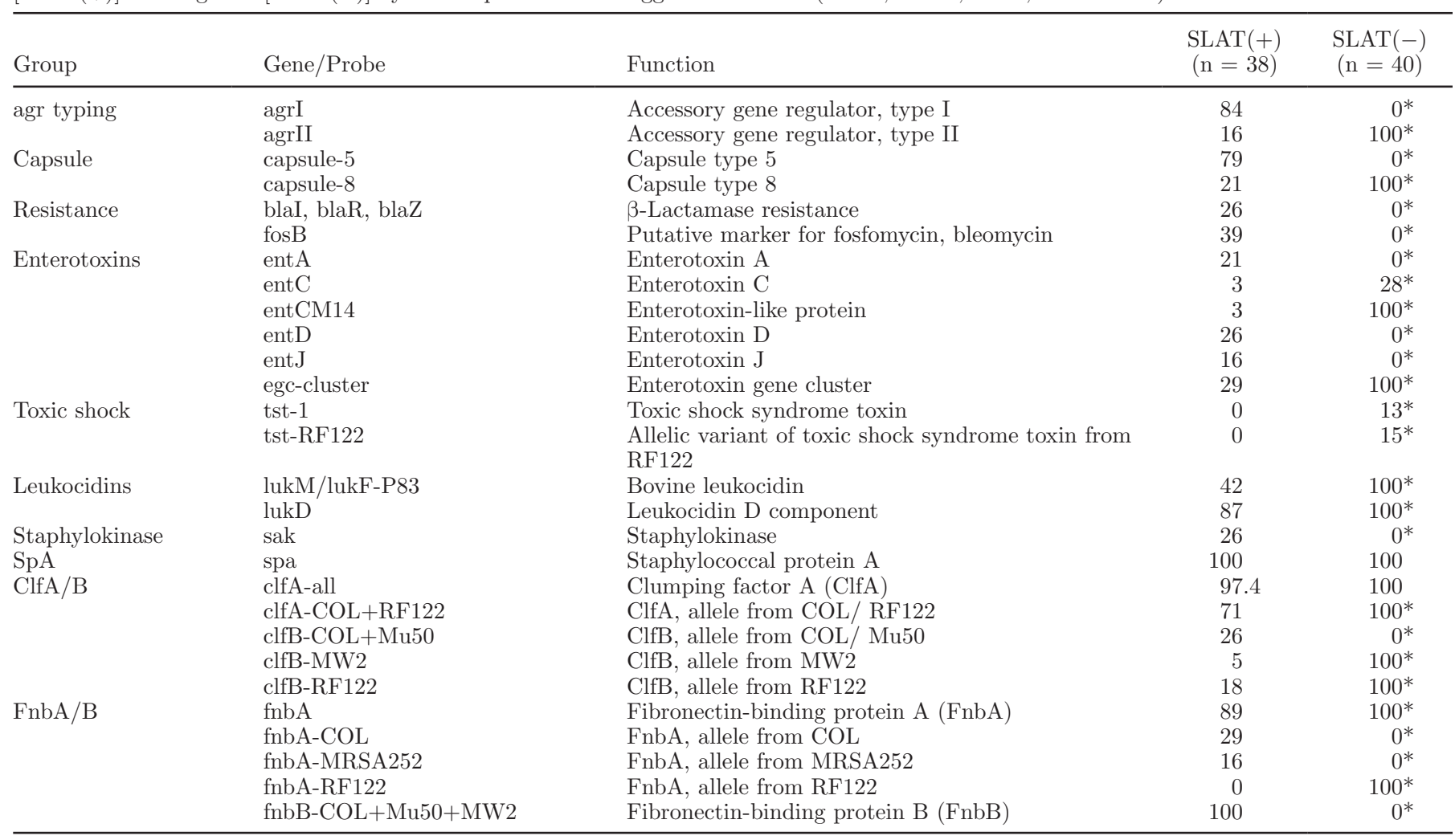

*Prevalence differed significantly between the SLAT $(+)$ and SLAT $(-)$ bovine Staph. aureus isolates tested in this study $(P<0.05)$.

exhibiting vanB, one of the genes involved in vancomycin resistance, was found to be sensitive to vancomycin by Etest (bioMérieux, La Balme les Grottes, France).

The DNA microarray results indicated that the resistance and virulence gene profiles of the SLAT $(-)$ strains in our study were very similar, but they differed largely from those of SLAT $(+)$ strains (Figure 1). Epidemiological studies suggest that a subset of Staph. aureus strains exhibits a distinctive genetic background that renders these strains highly successful in causing bovine mastitis (Herron-Olson et al., 2007). The SLAT(-) isolates characterized in our study lacked several virulence genes frequently found among SLAT $(+)$ isolates, including genes coding for resistance factors, staphylokinase, and enterotoxins A, $\mathrm{D}$, and J. This particular combination of enterotoxin genes (entA, entD, and entJ) was recently described to represent one of the main criteria in the identification of Staph. aureus strains classified as genotype B (Boss et al., 2011). Strains of this genotype were reported to be exclusively associated with very high (up to $65 \%$ ) within-herd prevalence of mastitis (Graber et al., 2009). In our study, strains exhibiting the combination of entA, entD, and entJ were assigned to $\mathrm{CC} 8$ only. A wide variety of enterotoxin genes can be found among
Staph. aureus isolates obtained from cases of bovine mastitis (Monecke et al., 2007). Still, the exact role of enterotoxins in the pathogenesis of bovine mastitis remains poorly understood (Haveri et al., 2007).

Among SLAT(-) strains, we found significantly higher numbers of several other virulence genes, including genes coding for toxic shock syndrome toxins (tst-1, tst-RF122), enterotoxins (entC, egc-cluster), and leukocidins (lukM/lukF-P83, lukD). Recent studies suggest that lukM/lukF-P83 plays a role in the pathogenesis of bovine mastitis (Barrio et al., 2006; Schlotter et al., 2012).

As false-negative results in the Staphaurex test are caused by impaired functionality of one or several of the targeted virulence factors SpA, ClfA/B, and FnbA/B, SLAT( $(-)$ strains are thought to be less virulent than SLAT $(+)$ strains (Stutz et al., 2011). The DNA microarray results revealed considerable heterogeneity regarding $c l f A / B$ and $F n b A / B$, and we found that $\operatorname{SLAT}(-)$ and SLAT $(+)$ isolates exhibited different alleles of the respective genes. Virulence factors ClfA/B and $\mathrm{FnbA} / \mathrm{B}$ represent a group of bacterial surface proteins designated "microbial surface components recognizing adhesive matrix molecules" (MSCRAMM). These proteins are of particular interest in the develop- 
ment of vaccines because they mediate adherence of Staph. aureus to components of the host's extracellular matrix. Attachment of the organism to the epithelial cells of the udder, as well as subsequent invasion of epithelial and endothelial cells was shown to depend on MSCRAMM (Lammers et al., 1999; Sinha et al., 1999), which also play a role in the evasion of host immune response (Higgins et al., 2006).

\section{CONCLUSIONS}

The genomic background of SLAT(-) and SLAT $(+)$ strains differed significantly with respect to a vast range of genes encoding crucial virulence and resistance factors. In consideration of the high heterogeneity among MSCRAMM genes detected by DNA microarray, we conclude that a combination of diverse antigens is crucial to the development of highly functional adhesin-based diagnostic tools and vaccines. Based on the comparison of microarray profiles and resistance phenotypes of SLAT $(+)$ and $\operatorname{SLAT}(-)$ bovine mastitis isolates investigated in this study, we hypothesize that SLAT $(+)$ strains exceed SLAT $(-)$ strains in virulence potential.

\section{ACKNOWLEDGMENTS}

This study was financed in part by a fellowship from the Swiss National Science Foundation (PBZHP3-138695).

\section{REFERENCES}

Barrio, M. B., P. Rainard, and G. Prévost. 2006. LukM/LukF'-PV is the most active Staphylococcus aureus leukotoxin on bovine neutrophils. Microbes Infect. 8:2068-2074.

Boss, R., J. Naskova, A. Steiner, and H. U. Graber. 2011. Mastitis diagnostics: Quantitative PCR for Staphylococcus aureus genotype B in bulk tank milk. J. Dairy Sci. 94:128-137.

CLSI (Clinical and Laboratory Standards Institute). 2008. Performance Standards for Antimicrobial Disk and Dilution Susceptibility Tests for Bacteria Isolated From Animals; Approved Standard M31-A3. 3rd ed. CLSI, Wayne, PA.

Fluit, A. C. 2012. Livestock-associated Staphylococcus aureus. Clin. Microbiol. Infect. 18:735-744. http://dx.doi.org/10.1111/j.14690691.2012.03846.x.

Graber, H. U., J. Naskova, E. Studer, T. Kaufmann, M. Kirchhofer, M. Brechbühl, W. Schaeren, A. Steiner, and C. Fournier. 2009. Mastitis-related subtypes of bovine Staphylococcus aureus are characterized by different clinical properties. J. Dairy Sci. 92:1442-1451.

Haveri, M., A. Roslöf, L. Rantala, and S. Pyöräla. 2007. Virulence genes of bovine Staphylococcus aureus from persistent and nonper- sistent intramammary infections with different clinical characteristics. J. Appl. Microbiol. 103:993-1000.

Herron-Olson, L., J. R. Fitzgerald, J. M. Musser, and V. Kapur. 2007. Molecular correlates of host specialization in Staphylococcus aureus. PLoS ONE 2:e1120.

Higgins, J., A. Loughman, K. P. M. van Kessel, J. A. G. van Strijp, and T. J. Foster. 2006. Clumping factor A of Staphylococcus aureus inhibits phagocytosis by human polymorphonuclear leucocytes. FEMS Microbiol. Lett. 258:290-296.

Huber, H., S. Koller, N. Giezendanner, R. Stephan, and C. Zweifel. 2010. Prevalence and characteristics of methicillin-resistant Staphylococcus aureus in humans in contact with farm animals, in livestock, and in food of animal origin, Switzerland, 2009. EuroSurveillance 15(16):pii:19542.

Huson, D. H., and D. Bryant. 2006. Application of phylogenetic networks in evolutionary studies. Mol. Biol. Evol. 23:254-267.

Klein, R. C., M. H. Fabres-Klein, M. A. Brito, L. G. Fietto, and A. D. Ribon. 2012. Staphylococcus aureus of bovine origin: Genetic diversity, prevalence and the expression of adhesin-encoding genes. Vet. Microbiol. 160:183-188. http://dx.doi.org/10.1016/j. vetmic.2012.05.025.

Lammers, A., P. J. M. Nuijten, and H. E. Smith. 1999. The fibronectin binding proteins of Staphylococcus aureus are required for adhesion to and invasion of bovine mammary gland cells. FEMS Microbiol. Lett. 180:103-109.

Monecke, S., P. Kuhnert, H. Hotzel, P. Slickers, and R. Ehricht. 2007. Microarray based study on virulence-associated genes and resistance determinants of Staphylococcus aureus isolates from cattle. Vet. Microbiol. 125:128-140.

Saini, V., J. T. McClure, D. T. Scholl, T. J. DeVries, and H. W. Barkema. 2012. Herd-level association between antimicrobial use and antimicrobial resistance in bovine mastitis Staphylococcus aureus isolates on Canadian dairy farms. J. Dairy Sci. 95:1921-1929.

Sakwinska, O., D. Morisset, J.-Y. Madec, A. Waldvogel, P. Moreillon, and M. Haenni. 2011. Link between genotype and antimicrobial resistance in bovine mastitis-related Staphylococcus aureus strains, determined by comparing Swiss and French isolates from the Rhône Valley. Appl. Environ. Microbiol. 77:3428-3432.

Schlotter, K., R. Ehricht, H. Hotzel, S. Monecke, M. Pfeffer, and K. Donat. 2012. Leukocidin genes lukf-P83 and lukM are associated with Staphylococcus aureus clonal complexes 151, 479 and 133 isolated from bovine udder infections in Thuringia, Germany. Vet. Res. 43:42 http://dx.doi.org/10.1186/1297-9716-43-42.

Sinha, B., P. P. François, O. Nüsse, M. Foti, O. M. Hartford, P. Vaudaux, T. J. Foster, D. P. Lew, M. Herrmann, and K. H. Krause. 1999. Fibronectin-binding protein acts as Staphylococcus aureus invasin via fibronectin bridging to integrin $\alpha 5 \beta 1$. Cell. Microbiol. $1: 101-117$

Steeneveld, W., T. van Werven, H. W. Barkema, and H. Hogeveen. 2011. Cow-specific treatment of clinical mastitis: An economic approach. J. Dairy Sci. 94:174-188.

Stutz, K., R. Stephan, and T. Tasara. 2011. SpA, ClfA, and FnbA genetic variations lead to Staphaurex test-negative phenotypes in bovine mastitis Staphylococcus aureus isolates. J. Clin. Microbiol. 49:638-646.

Wattinger, L., R. Stephan, F. Layer, and S. Johler. 2012. Comparison of Staphylococcus aureus isolates associated with food intoxication with isolates from human nasal carriers and human infections. Eur. J. Clin. Microbiol. Infect. Dis. 31:455-464.

Wells, S. J., S. L. Ott, and A. H. Seitzinger. 1998. Key health issues for dairy cattle - New and old. J. Dairy Sci. 81:3029-3035. 